\title{
Simultaneous Boundary and Partial Volume Estimation in Medical Images
}

\author{
Dzung L. Pham and Pierre-Louis Bazin \\ Laboratory of Medical Image Computing, Johns Hopkins University, Baltimore, MD 21224
}

\begin{abstract}
Partial volume effects are present in nearly all medical imaging data. These artifacts blur the boundaries between different regions, making accurate delineation of anatomical structures difficult. In this paper, we propose a method for unsupervised estimation of partial volume fractions in single-channel image data. Unlike previous methods, the proposed algorithm simultaneously estimates partial volume fractions, the means of the different tissue classes, as well as the the locations of tissue boundaries within the image. The latter allows the partial volume fractions to be constrained to represent pure or nearly pure tissue except along tissue boundaries. We demonstrate the application of the algorithm on simulated and real magnetic resonance images.
\end{abstract}

\section{Introduction}

Segmentation of three-dimensional volumetric images is an important goal in many medical imaging applications such as in the localization of pathology, quantification, and computer integrated surgery. Because of the finite resolution of imaging devices, however, nearly all images suffer from partial volume effects. These artifacts result when different tissue classes in the image contribute to a single voxel, thereby causing boundaries to be blurred and ambiguously defined. As a result, standard voxel-based segmentation techniques that do not address partial volume effects often fail to capture fine details that may be present in the original image. In many applications, this preservation of fine details can be critical in the quantification and analysis of anatomical structures and pathology.

In tissue classification methods, the most common approach to dealing with partial volume effects is to obtain a soft or fuzzy segmentation. Rather than exclusively classifying a voxel as belonging to a particular class, soft segmentation methods allow for a continuous grade of membership within different classes. These memberships can be computed using fuzzy clustering [12] or probabilistic classification alorithms [3]4]. This approach, however, does not explicitly model partial volume effects and can therefore be susceptible to certain artifacts in the resulting segmentation [5]. Rather than measure the partial volume content, some methods have attempted to segment out partial volume voxels as separate classes [6]. Recent work has focused on directly estimating partial volume fractions in multi-channel [7/89] and single-channel images [5 10]11]. Because of the ill-posed nature of the problem, a fundamental difficulty in most of these partial volume estimation methods has been how to incorporate appropriate prior information into the estimation framework. 
In this paper, we incorporate boundary information into the estimation procedure. Tissue boundaries within the image play a key role in partial volume effects because they, in fact, define where partial voluming will occur. Knowledge of the boundary locations allows us to constrain the partial volume fractions to be smooth and to represent pure tissue away from boundaries. The overall framework for estimating partial volume fractions and boundaries is based on an approximation of the Mumford-Shah functional. This leads to an iterative algorithm for estimation of the partial volume fractions, mean intensities of the tissue classes, and the boundaries. A multigrid algorithm is used to solve the discretized partial differential equation in the boundary estimation. This paper builds upon our previous work presented in [12,5].

\section{Background}

In [13] and [14], segmentation of two-dimensional magnetic resonance (MR) images was performed based on the minimization of the following energy functional with respect to the segmentation $f$, and the boundary field $b$ :

$$
\iint_{\Omega} \alpha(y-f)^{2}+\beta(1-b)^{2}|\nabla f|^{2}+\gamma\left(\rho|\nabla b|^{2}+\frac{1}{\rho} s^{2}\right) d x d y
$$

The above equation closely resembles the well-studied Mumford-Shah functional [13], except it uses a continuously-valued boundary function rather than a binary one. In this equation, $\Omega$ is the domain of the image, $y$ is the image data, and $\alpha, \beta, \gamma$, and $\rho$ are weighting parameters controlling the balance of each term. The estimated image $f$ is an approximation of $y$ and not a true segmentation or tissue classification. It will be piecewise smooth, as dictated by the second term that penalizes the gradient of $f$ where the boundary field is small. The boundary field at each pixel possesses a value between zero and one and will be large where $f$ is discontinuous. The third term penalizes both the existence of boundaries, as well as the gradient of the boundary field. The effectiveness of equation (1) stems from the fact that the $f$ is smoothed within but not across boundaries, and because the boundary field itself is constrained to be smooth.

\section{Energy Formulation}

In this section, it is shown how the edge-adaptive properties of 11 can be incorporated into a partial volume estimation algorithm. Three major modifications of 11 are made. First, the approximate image $f$ is replaced with a partial volume image representation:

$$
f_{j}=\sum_{k=1}^{C} s_{j k} c_{k}
$$

where $s_{j k}$ is the partial volume fraction at voxel $j$ for class $k$, and $c_{k}$ is the representative tissue class intensity for class $k$. The summation over classes on $s_{j k}$ is restricted to be equal to one and we will refer to $c_{k}$ as the class mean. Second, instead of penalizing the gradient of the partial volume fractions, we employ a penalty function that constrains the 
partial volumes to be pure or nearly pure away from boundaries. Third, the boundary field is allowed to exist between voxel locations on a complementary grid and is decomposed into its directional components. For notational simplicity in the equations that follow, we focus on the single channel two-dimensional case, but the generalization to three dimensions and multiple channels is straightforward.

We propose to minimize the following energy functional with respect to the partial volume fractions $s$, the mean intensities $c$, and the boundary field $b$ :

$$
\begin{aligned}
E & =\alpha \sum_{j \in \Omega}\left\|y_{j}-\sum_{k=1}^{K} s_{j k} c_{k}\right\|^{2} \\
& +\frac{\beta}{2} \sum_{j \in \Omega} \sum_{k=1}^{K} \sum_{m \neq k}\left(\sum_{l \in N_{j}^{(x)}}\left(1-b_{j \mid l}^{(x)}\right)^{2} s_{j k} s_{l m}+\sum_{l \in N_{j}^{(y)}}\left(1-b_{j \mid l}^{(y)}\right)^{2} s_{j k} s_{l m}\right) \\
& +\frac{\gamma}{2} \sum_{j \in \Omega_{x}}\left(\frac{1}{\rho}\left(b_{j}^{(x)}\right)^{2}+\rho \sum_{l \in N_{j}}\left(b_{j}^{(x)}-b_{l}^{(x)}\right)^{2}\right)+\frac{\gamma}{2} \sum_{j \in \Omega_{y}}\left(\frac{1}{\rho}\left(b_{j}^{(y)}\right)^{2}+\rho \sum_{l \in N_{j}}\left(b_{j}^{(y)}-b_{l}^{(y)}\right)^{2}\right)
\end{aligned}
$$

Although Eq. 2]may appear complicated, it can be broken down into fairly simple parts. The first term follows the standard linear assumption used in most partial volume estimation methods.

The second line of Eq. 2 is based on the smoothness penalty on partial volume fractions proposed in [5]. This penalty is similar to the Potts model used in Markov random field theory and enforces two constraints: 1) the partial volume should be similar to its neighbors; 2) the partial volume should be close to zero or one. The main difference is that the penalty is now modulated by horizontal and vertical boundary fields, $b^{(x)}$ and $b^{(y)}$. Thus, the penalty function is relaxed at voxels near boundaries. We use the notation $N_{j}^{(x)}$ and $N_{j}^{(y)}$ to denote the set of horizontal and vertical neighbors of $j$, and $b_{j \mid l}$ to denote the boundary value between pixel locations $j$ and $l$.

The third line of Eq. 2 consists of penalty functions on the horizontal and vertical boundary fields to ensure that they are also smooth. This penalty function is the same as the boundary penalty functions utilized in [15] except that the boundary field has been decomposed into separate horizontal and vertical components. The complementary horizontal and vertical grids of the boundary fields are denoted $\Omega_{x}$ and $\Omega_{y}$, respectively. It is possible to also consider diagonal boundaries at the cost of greater computational expense. The parameters $\alpha, \beta, \gamma$, and $\rho$ control the relative balance of each term. For the boundary field, $\gamma$ controls the magnitude of each field while $\rho$ controls the overall smoothness. Figure 1 shows the boundary fields estimated from a synthetic image with some additive noise. The estimated partial volumes are used to construct a hard classification by assigning each pixel to the class with the greatest partial volume contribution, as shown in Figure 1(b). Figures 1 (c)-(d) shows the horizontal and vertical boundary fields estimated on the complimentary grid. By increasing $\rho$, it is possible to diffuse the boundary field to a greater extent, as shown in Figure 1e). Note that the diffusion is symmetric. 


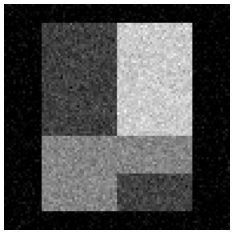

(a)

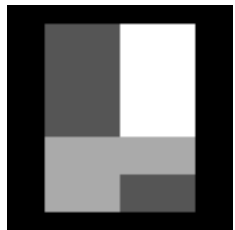

(b)

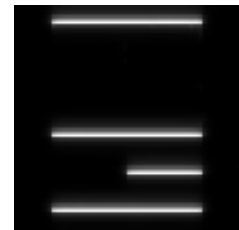

(c)

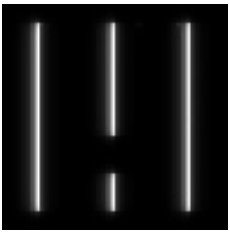

(d)

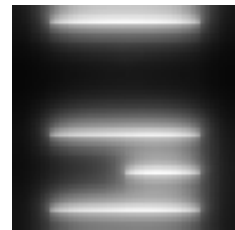

(e)

Fig. 1. Illustration of boundary fields: (a) input image, (b) classification result, (c) horizontal boundary field, (d) vertical boundary field, (e) horizontal boundary field with increased $\rho$ parameter.

\section{Algorithm}

To minimize the energy function, a coordinate descent method is employed. The algorithm iteratively seeks a zero gradient condition of (2). For single-channel images, we employ the simplifying assumption that only two classes can contribute to a single voxel. To improve readability, we present the equations for $b_{j}$ on the standard grid. It is straightforward to modify the equations such that the neighborhoods are decomposed to consider horizontal and vertical boundaries. The basic computations are the same.

If $a$ and $b$ denote the two contributing classes to a voxel $j$, the estimator for the partial volume fraction is derived by taking the first derivative of (2) with respect to $s_{j a}$ and setting it to zero. This yields

$$
s_{j a}=\frac{\left(c_{b}-y_{j}\right)\left(c_{b}-c_{a}\right)+\beta\left(1-b_{j}\right)^{2} \sum_{i \in N_{j}}\left(\sum_{m \neq b} s_{l m}-\sum_{m \neq a} s_{l m}\right)}{\left(c_{b}-c_{a}\right)^{2}}
$$

Values of $s_{j a}$ are restricted to lie between zero and one and we sort the mean intensities such that $c_{b}>c_{a}$. The value of $s_{j b}$ is computed as $1-s_{j a}$.

A necessary condition on $b_{j}$ for (2) to be minimized is

$$
\sum_{l \in N_{j}} \sum_{k=1}^{K} \sum_{m \neq k} s_{j k} s_{l m}=\left(\sum_{l \in N_{j}} \sum_{k=1}^{K} \sum_{m \neq k} s_{j k} s_{l m}+\frac{\gamma}{\rho \beta}\right) b_{j}+\frac{\gamma \rho}{\beta} \nabla^{2} b_{j}
$$

Equation (4) is a difference equation with spatially varying coefficients. A multigrid algorithm is employed to efficiently determine a solution [2].

The class means are computed iteratively using the following equation:

$$
c_{k}=\frac{\sum_{j \in \Omega} s_{j k}\left(y_{j}-\sum_{l \neq k} s_{j l} c_{l}\right)}{\sum_{j \in \Omega} s_{j k}^{2}} .
$$

Our algorithm can be summarized as follows:

1. Obtain the initial class means.

2. Estimate the spread coefficients using the following procedure:

a) Compute (3) for $\left(\begin{array}{l}K \\ 2\end{array}\right)$ combinations of $a$ and $b$. 
b) Select partial volume configuration with minimal energy.

c) Repeat 3(a) \& 3(b) for all voxels in the image.

3. Estimate the boundary field using (4).

4. Estimate the class means 5 .

5. Repeat 2-4 until convergence.

Convergence is assumed to be achieved when the maximum change over all spread coefficients between iterations is less than 0.01. To improve stability of the algorithm, we employ a simple relaxation procedure that weights the current estimate with the previous estimate. This relaxation is eventually removed as iterations are increased and the algorithm nears convergence.

\section{Gain Field Correction for MR Images}

MR images can suffer from signal inhomogeneities, causing smooth intensity variations over the image space. This effect is usually represented by a multiplicative gain field [3]. This can be modeled by placing an additional variable, $g_{j}$, within the first term of 2 .

$$
\sum_{j \in \Omega}\left\|y_{j}-g_{j} \sum_{k=1}^{K} s_{j k} c_{k}\right\|^{2}
$$

We model the gain field as a 2D polynomial function of low degree (degree 3 or 4 usually provides enough flexibility):

$$
g_{j}=\sum_{n, m=1}^{N, M} v_{n, m} P_{n, m}(j),
$$

where $P_{n, m}$ is a polynomial basis function of degree $n$ in the horizontal and $m$ in the vertical directions. We choose Chebyshev polynomials for their numerical stability and their properties for function approximation [16].

The optimal polynomial coefficients $v_{n, m}$ are the solution of the least-squares equation:

$$
\sum_{j \in \Omega}\left\|y_{j}-\sum_{n, m=1}^{N, M} v_{n, m} P_{n, m}(j) \sum_{k=1}^{C} s_{j k} c_{k}\right\|^{2}=0
$$

In matrix notations, we can rewrite the above equation as $(Y-S \cdot P \cdot V)^{T}(Y-S$. $P \cdot V)=0$, with vectors $Y=\left[y_{j}\right], V=\left[v_{n, m}\right]$ and matrices $P=\left[P_{n, m}(j)\right]$ and $S$ is a diagonal matrix with central elements $\sum_{k=1}^{C} s_{j k} c_{k}$. The solution is given by:

$$
V=\left(P^{T} C P\right)^{-1} P^{T} Y
$$

To reduce the computational burden of estimating for all unknown variables simultaneously, we solve the gain field in an initialization step using the simplified model described in [5]. This model does not use a spatial prior and assumes that the contributing tissue classes for a voxel are the classes whose centroids bound the intensity value of that voxel. 


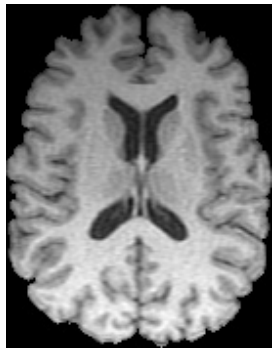

(a)

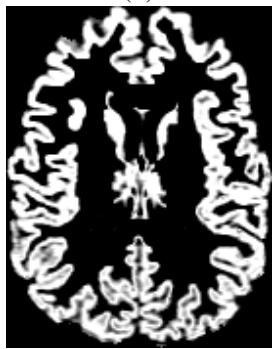

(d)

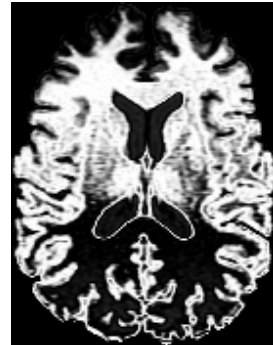

(b)

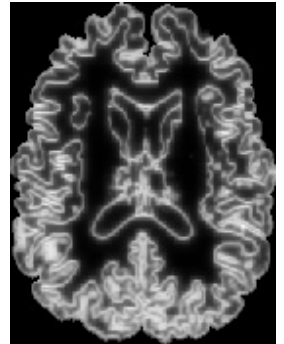

(e)

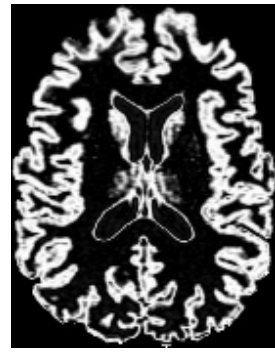

(c)

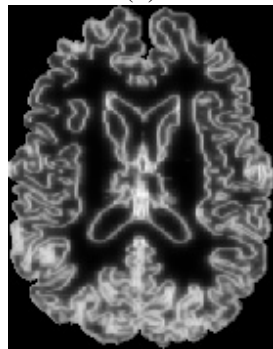

(e)

Fig. 2. (a) Actual MR image with simulated inhomogeneity, (b) fuzzy $c$-means gray matter segmentation, (c) enhance fuzzy c-means result, (d) partial volume result, (e)-(f) horizontal and vertical boundary fields.

\section{Results}

Figure 2] compares the results of the partial volume estimation algorithm with the standard fuzzy $c$-means algorithm, and an enhanced fuzzy clustering algorithm that incorporates gain field and noise correction [17]. Figure 2]a) is a slice from a real MR image data set. A gain field has been artificially applied to the image to increase the difficulty of the segmentation. The standard fuzzy $c$-means algorithm yields rather poor results, while the partial volume and enhanced fuzzy clustering algorithm provide reasonable results. The partial volume result has a reduced rim artifact around the ventricle but possesses thicker gray matter. The boundary fields illustrate that most of the structure within the boundary field is not purely horizontal or vertical. For this example, the following parameter settings were used: $\alpha=1, \beta=100, \gamma=100$, and $\rho=1$. The relaxation weight was initially set to 0.25 and incremented by 0.25 until equal to one. A third degree polynomial was used to the model the inhomogeneity field.

Figure 3 shows the results of our algorithm when applied to the Brainweb phantom from McGill University [18]. The image was simulated with 5\% noise and 40\% inhomogeneity. The results of the algorithm very closesly follow the true partial volume fractions used to generate the synthetic image. The estimated fractions are mostly free from the effects of the noise. In addition, the estimated gain field captures the darkening of intensities seen at the bottom of the image. For this example, the following parameter settings were used: $\alpha=1, \beta=150, \gamma=100, \rho=1$, 3rd degree polynomial for the inhomogeneity field.. 


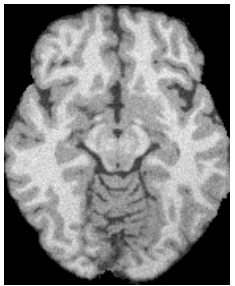

(a)

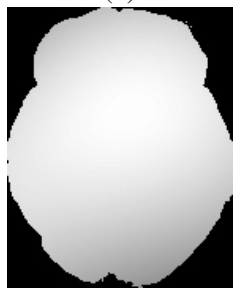

(f)

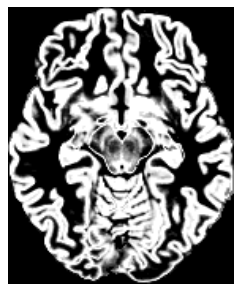

(b)

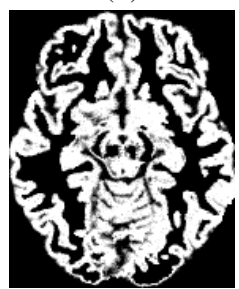

(g)

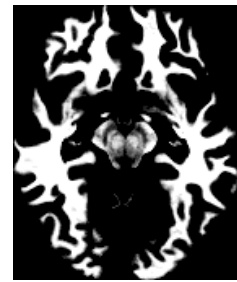

(c)

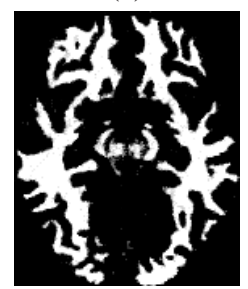

(h)

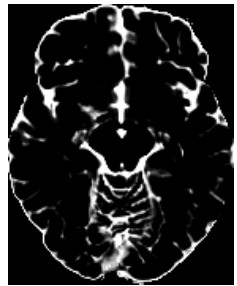

(d)

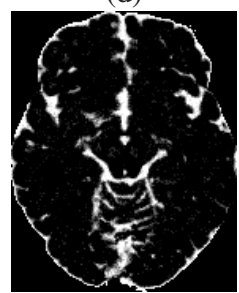

(i)

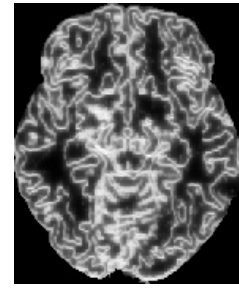

(e)

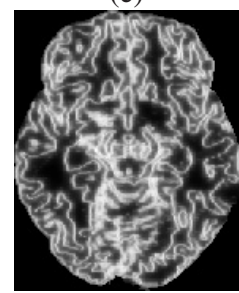

(j)

Fig. 3. Brainweb phantom image: (a) $T_{1}$-weighted image, (b)-(d) true gray matter, white matter, and cerebrospinal fluid partial volumes, field, (e) horizontal boundary field, (f) estimated gain field, (g)-(i) estimated partial volume fractions, (j) vertical boundary field.

\section{Discussion}

We have proposed a new approach for partial volume estimation that simultaneously computing a boundary field. The boundary field allows the partial volume fractions to be smooth and represent pure tissue at pixels that are away from the boundaries. A subtle benefit of this approach is that in our previous approach [5], a prior probability function on the tissue class means was required to stabilize the estimation. This is no longer needed because the boundary field allows the segmentation to be essentially binary or "hard" everywhere except at boundaries, thereby making it more stable. We have found that convergence is still rather slow however. Execution times for two-dimensional images were on the order of a couple of minutes on Pentium IV 3.0 GHz, with most of the time devoted to the solution of the boundary fields. Although the multigrid solution is efficient, hundreds of iterations are usually required for the overall algorithm to converge. In future work, we plan to investigate improving the convergence properties of the algorithm and perform further validation of its performance. We have found that gray matter in brain images is perhaps overestimated. This can be corrected by using separate variances for different class configurations, similar to mixture model approaches.

\section{References}

1. R.C. Herndon, J.L. Lancaster, J.N. Giedd, and P.T. Fox. Quantification of white matter and gray matter volumes using T1 parametric images using fuzzy classifiers. J. Magnetic Resonance Imaging, 6:425-435, 1996.

2. D.L. Pham and J.L. Prince. Adaptive fuzzy segmentation of magnetic resonance. IEEE Trans. Med. Imag., 18:737-752, 1999. 
3. W.M. Wells, W.E.L. Grimson, R. Kikins, and F.A. Jolesz. Adaptive segmentation of MRI data. IEEE Trans. Med. Imag., 15:429-442, 1996.

4. K.V. Leemput, F. Maes, D. Vandermulen, and P. Seutens. Automated model-based tissue classification of MR images of the brain. IEEE Trans. Med. Imag., 18(10):897-908, 1999.

5. D.L. Pham and J.1. Prince. Unsupervised partial volume estimation in single-channel image data. In Proceedings of the IEEE Workshop on Mathematical Methods in Biomedical Image Analysis, pages 170-177, Hilton Head, SC, June 11-12 2000.

6. P. Santago and H.D. Gage. Quantification of MR brain images by mixture density and partial volume modeling. IEEE Trans. Medical Imaging, 12:566-574, 1993.

7. H.S. Choi, D.R. Haynor, and Y. Kim. Partial volume tissue classification of multichannel magnetic resonance images - a mixel model. IEEE Trans. Medical Imaging, 10:395-407, 1991.

8. H. Soltanian-Zadeh, J.P. Windham, D.J. Peck, and A.E. Yagle. Optimal transformation for correcting partial volume averaging effects in magnetic resonance imaging. IEEE Trans. Nucl. Sci., 11:302-318, 1992.

9. D.H. Laidlaw, K.W. Fleischer, and A.H. Barr. Partial-volume bayesian classification of material mixtures in MR volume data using voxel histograms. IEEE Trans. Med. Imag., 17:98-107, 1998.

10. D.W. Shattuck, S.R. Sandor-Leahy, K.A. Schaper, D.A. Rottenberg, and R.M. Leahy. Magnetic resonance image tissue classification using a partial volume model. Neuroimage, 13:856-876, 2001.

11. K. Van leemput, F. Maes, D. Vandermeulen, and P. Suetens. A unifying framework for partial volume segmentation of brain mr images. IEEE Trans. Med. Imag., 22:105 - 119, 2003.

12. D.L. Pham. Unsupervised tissue classification in medical images using edge-adaptive clustering. In Proceedings of the 25th International Conference of the IEEE Engineering in Medicine and Biology Society, Cancun, Mexico, Sept. 17-21 2003.

13. H.H. Pien and J.M. Gauch. Variational segmentation of multi-channel MRI images. In Proceedings of the 1994 IEEE International Conference on Image Processing, volume 3, pages 508-512, 1994.

14. J. Kaufhold, M. Schneider, A.S. Willsky, and W.C. Karl. A statistical method for efficient segmentation of MR imagery. Internation Journal of Pattern Recognition and Artificial Intelligence, 11(8):1213-1231, 1997.

15. J.M. Gauch J. Shah, H.H. Pien. Recovery of surfaces with discontinuities by fusing shaing and range data within a variational framework. IEEE Trans. on Image Processing, 5(8):12431251, 1996.

16. Philip J. Davis. Interpolation and Approximation. Dover, 1975.

17. D.L. Pham. Robust fuzzy segmentation of magnetic resonance images. In Proceedings of the 14th IEEE Symposium on Computer-Based Medical Systems, pages 127-131, Bethesda, MD, July 26-27 2001.

18. D.L. Collins, A.P. Zijdenbos, V. Kollokian, J.G. Sled, N.J. Kabani, et al. Design and construction of a realistic digital brain phantom. IEEE Trans. Med. Imag., 17:463-468, 1998. 